\title{
A Prospective Study of Lupus and Rheumatoid Arthritis in Relation to Deployment in Support of Iraq and Afghanistan: The Millennium Cohort Study
}

\author{
Kelly A. Jones, ${ }^{1}$ Nisara S. Granado, ${ }^{1}$ Besa Smith, ${ }^{1}$ Donald J. Slymen, ${ }^{2}$ Margaret A. K. Ryan, ${ }^{3}$ \\ Edward J. Boyko, ${ }^{4}$ Gary D. Gackstetter, ${ }^{5}$ Christopher J. Phillips, ${ }^{1}$ and Tyler C. Smith ${ }^{1}$ \\ ${ }^{1}$ Deployment Health Research Department, Naval Health Research Center, 140 Sylvester Road, San Diego, CA 92106-3521, USA \\ ${ }^{2}$ Graduate School of Public Health, San Diego State University, San Diego, CA 92182-4162, USA \\ ${ }^{3}$ Occupational Health Department, Naval Hospital Camp Pendleton, Camp Pendleton, CA 92055, USA \\ ${ }^{4}$ Department of Veterans Affairs Puget Sound Health Care System, Seattle Epidemiologic Research and Information Center, Seattle, \\ WA 98108-1532, USA \\ ${ }^{5}$ Analytic Services, Inc. (ANSER), Arlington, VA 22206-2233, USA
}

Correspondence should be addressed to Kelly A. Jones, kelly.jones@med.navy.mil

Received 13 June 2011; Accepted 24 August 2011

Academic Editor: Maria Bokarewa

Copyright () 2011 Kelly A. Jones et al. This is an open access article distributed under the Creative Commons Attribution License, which permits unrestricted use, distribution, and reproduction in any medium, provided the original work is properly cited.

The objective of this study was to prospectively assess the association between deployment in support of the operations in Iraq and Afghanistan and newly reported lupus and rheumatoid arthritis while also considering the effects of demographic, behavioral, and occupational characteristics. A total of 77,047 (2001-2003) and 31,110 (2004-2006) participants completed the baseline Millennium Cohort questionnaire and were resurveyed approximately every 3 years. Longitudinal analyses were used to assess the adjusted association between deployment to Iraq and Afghanistan with and without combat exposures and newly reported disease. After adjusting, deployment was not significantly associated with newly reported lupus compared with nondeployers. However, compared with nondeployers, deployers with and without combat exposures were significantly less likely to newly report rheumatoid arthritis. Women, non-Hispanic black, and Hispanic participants had a significantly elevated risk for both diseases. Overall, deployment was not associated with an increased risk of newly reported lupus or rheumatoid arthritis.

\section{Introduction}

Representing a diverse family of chronic disease, autoimmune diseases are disabling and have a variety of natural histories. It is estimated that rheumatoid arthritis (RA) and systemic lupus erythematosus (SLE) affect considerable numbers of US residents (460 per 100,000 persons and 40-150 per 100,000, resp.) [1-5]. The etiologies of these conditions are unknown, and both diseases cause chronic, systemic inflammation resulting in tissue and/or organ damage [6]. Several studies document how these diseases adversely affect the general health of many in the US population [2-5]; however, research is sparse in the US military population on not only the frequency but also the risk factors associated with their occurrence.
Military personnel deployed to combat regions during the current operations in Iraq and Afghanistan are often exposed to stressful situations putting this population at higher risk for stress-related medical conditions and lifestyle choices associated with poorer health outcomes [7-11]. There have been concerns with previous military operations that service members potentially have a higher risk of SLE and RA due to traumatic stressors experienced during deployment [12-14]. Furthermore, military personnel may experience several environmental and occupational exposures, including pesticides (environmental and topical), paints, solvents, and silica dust that have been associated with SLE and RA in the general US population [1, 15-21]. It is important to understand potential factors that may increase the risk of these complex diseases, especially when military 
deployments may include unique environmental and occupational exposures [10, 22]. Using data from the Millennium Cohort Study, these medical outcomes were prospectively investigated in relation to demographic, occupational, and behavioral characteristics.

\section{Materials and Methods}

2.1. Study Population. Participants were from the Millennium Cohort Study, a prospective, population-based sample consisting of a weighted random sample of all US service members on rosters as of October 2000 and October 2003, who were enrolled beginning in 2001 and 2004, respectively. The Cohort was primarily designed to evaluate long-term health effects related to military service over a 21-year time period. This study includes participants from the 2001 and 2004 enrollment cycles. A more detailed description of the Millennium Cohort Study has been published elsewhere [2325].

For the 2001 enrollment, a total of 77,047 participants voluntarily consented and completed the baseline questionnaire (2001-2003), with 63,372 completing at least one of the follow-up questionnaires (2004-2006, 2007-2008). For the 2004 enrollment, 31,110 consented and completed the baseline questionnaire (2004-2006) with 17,152 completing the first follow-up questionnaire (2007-2008). Of the 80,524 participants who completed the baseline and follow-up questionnaires, exclusions included missing or affirmative self-report of lupus or RA at baseline, missing responses to questions relating to lupus or RA on at least one followup questionnaire, self-reported disease at first follow-up but not at the second follow-up, self-reported disease prior to first deployment, and affirmatively responded to all providerdiagnosed disorders. This left a total population of 76,425 and 74,643 participants for the analyses of lupus and RA, respectively.

2.2. Data Collection. Fixed and time-varying demographic and military characteristics were obtained from the electronic personnel files maintained at the Defense Manpower Data Center and linked to each participant at baseline and each follow-up. Data included sex, birth year, race/ethnicity, education, marital status, branch of service, service component, military pay grade, military occupation, and deployment experience in support of the operations in Iraq and Afghanistan from 2001 to 2008.

Participants were defined as deployers at baseline if they had deployed for 1 or more days in support of operations in Iraq and Afghanistan prior to baseline and were defined as deployers at each follow-up if they were deployed for 1 or more days between follow-up survey assessments. Deployers were further classified as with or without combat experiences on each follow-up based on a positive self-report to personally witnessing or being exposed in the past 3 years to a person's death due to war or disaster, physical abuse, dead or decomposing bodies, maimed soldiers or civilians, or prisoners of war or refugees. Combat experiences were not assessed at baseline due to uncertainty of deploymentexposure relation because the question was addressed in the context of "ever" rather than the "past 3 years." Participants not meeting the deployment definition were considered nondeployers.

Using baseline and follow-up surveys, time-varying general health, behavioral, and occupational characteristics were included to evaluate whether certain subpopulations were more likely to newly report lupus or RA. General health was investigated using the physical and mental component summary scores from the Medical Outcomes Study Short Form 36-Item Health Survey for Veterans [26-31]. Participants' component scores were categorized into quartiles. Body mass index was classified as normal/underweight $\left(<25 \mathrm{~kg} / \mathrm{m}^{2}\right)$, overweight $\left(25\right.$ to $\left.<30 \mathrm{~kg} / \mathrm{m}^{2}\right)$, and obese $\left(\geq 30 \mathrm{~kg} / \mathrm{m}^{2}\right)$. Nonsmokers, past smokers, and current smokers were identified using survey questions addressing lifetime smoking of at least 100 cigarettes (5 packs), a successful attempt to quit smoking, and cigarette use in the past year. Alcohol misuse was evaluated using the CAGE questionnaire (cutting down, annoyance by criticism, guilty feeling, and eye openers) $[32,33]$. Finally, environmental and occupational exposures were derived from an affirmative self-report of ever being exposed prior to newly reporting disease to the following: chemical and biological warfare agents, occupational hazards requiring personal protective equipment; routine skin contact with paint, solvents, or substances, microwaves (excluding microwave ovens), and topical (creams, sprays, uniform treatments) and environmental pesticides.

2.3. Outcomes. Lupus and RA were investigated using the baseline survey question "Has your doctor or other health professional ever told you that you have any of the following conditions?" At follow-up, participants were asked the same question, but in the context of "in the last 3 years." Newly reported lupus and RA were defined as an affirmative selfreport to these diseases on either of the follow-up surveys among participants reporting no prior condition. Those who did not report an outcome of interest constituted the nondiseased control comparison groups.

2.4. Statistical Analyses. Descriptive and univariate analyses were conducted to examine unadjusted associations of the study outcomes with demographic, occupational, and behavioral risk factors. Separate models were developed for both newly reported lupus and RA. Longitudinal analyses were performed using the generalized estimating equations method to compare the adjusted association between deployment status and both outcomes [34, 35]. All analyses adjusted for time calculated as the number of years between baseline and each follow-up survey. A model analysis was performed using a variation inflation factor greater than four to indicate a potential problem with multicollinearity. Confounders were defined as variables changing the association between each disease outcome and deployment status by more than $10 \%$ [36]. Variables that were not confounders nor statistically significant in the model at $P$ $<0.05$ were manually removed using a backward reduction method to establish the final models. If a variable was neither confounding nor significant but was consistently associated with either outcome in previous published literature, the 
variable remained in the model. Statistical analyses were performed using SAS software version 9.2 (SAS Institute, Inc., Cary, NC, USA).

\section{Results}

3.1. Lupus. Baseline characteristics and deployment status stratified by whether participants reported lupus at followup are shown in Table 1. Over an average of 5.6 years between baseline and last follow-up, the cumulative incidence of lupus was 0.34 per 1000 person-years. Lupus was newly reported among $0.2 \%$ of nondeployers and $0.1 \%$ of deployers. Deployers were proportionately more likely to be male, younger, less educated, and in the Army compared with nondeployers.

The results from the univariate analysis and the final adjusted model are shown in Table 2. Subgroups proportionately more likely to newly report lupus were female, nonHispanic black and Hispanic, not married, and of lower mental and physical health. The following variables were removed from the full adjusted model (not shown), because they were not significant $(P>0.05)$ and did not confound the results: education, marital status, service component, pay grade, service branch, occupation, body mass index, smoking status, alcohol misuse and exposure to chemical and biological warfare agents, occupational hazards requiring personal protective equipment, skin contact with paints, solvents, or substances, microwaves (excluding microwave ovens), and topical and environmental pesticides. The final adjusted model revealed deployments with and without combat exposures were not significantly associated with newly reported lupus when compared with nondeployers $(P=0.37)$. Women were nearly twice as likely (adjusted odds ratio $(\mathrm{AOR})=1.81,95 \%$ confidence interval $[\mathrm{CI}]: 1.20-2.72$ ) to newly report lupus compared with men. Non-Hispanic black (AOR $=2.66,95 \%$ CI: $1.70-4.17)$ and Hispanic (AOR $=2.35$, 95\% CI: $1.27-4.36)$ participants had over two times the risk of newly reported lupus than non-Hispanic white participants. Those reporting low mental and physical health were at significantly higher risk of newly reported lupus when compared with those reporting high mental or physical health ( $>75$ th percentile).

3.2. Rheumatoid Arthritis. Baseline characteristics and deployment status stratified by whether participants reported RA at follow-up are shown in Table 1. The cumulative incidence of RA between 2001 and 2008 was 3.30 per 1000 person-years. RA was newly reported among $1.9 \%$ of nondeployers and $1.1 \%$ of deployers. Similar to lupus deployers were proportionately more likely to be male, younger, less educated, and in the Army compared with nondeployers.

Results for the univariate analysis and the final adjusted model are shown in Table 3. Subgroups proportionately more likely to newly report RA were female, non-Hispanic black and Hispanic, less educated, overweight, past and current smokers, alcohol misusers, of lower mental and physical health, having had unknown exposure to occupational hazards requiring personal protective equipment, and having had exposure to skin contact with paints, solvents, or substances; microwaves; and environmental or topical pesticides. To establish the final model, the following covariates were removed from the full model (not shown), because they were not significant $(P>0.05)$ and were not determined to be confounders: marital status, alcohol misuse, and exposure to occupational hazards requiring personal protective equipment, routine skin contact with paints, solvents, or substances, and environment and topical pesticides. The final adjusted model revealed those deployed with $(\mathrm{AOR}=0.74,95 \% \mathrm{CI}: 0.62-0.88)$ and without $(\mathrm{AOR}=$ $0.59,95 \%$ CI: $0.48-0.73$ ) combat exposures were significantly less likely to newly report RA. Those significantly less likely to newly report RA were younger; Reserve or National Guard; officers; Air Force, Navy, or Coast Guard; and health care specialists. Women were 1.26 (95\% CI: 1.08-1.47) times more likely to report RA than men. Non-Hispanic black $(\mathrm{AOR}=1.77,95 \% \mathrm{CI}: 1.51-2.08)$ and Hispanic $(\mathrm{AOR}=$ 1.40, 95\% CI: 1.11-1.76) participants had a significantly higher risk of disease compared with non-Hispanic white participants. Those with some college, obese respondents, and current and past smokers also had a significantly elevated risk of RA. Lower mental and physical health, exposure to chemical and biological warfare agents, and "do not know" response to exposure to microwaves were significantly associated with an increased risk of newly reported RA.

\section{Discussion}

Concern was raised after the 1991 Gulf War [13, 14] of a possible association between service in this conflict and a higher risk of SLE and RA. This potential association continues to be of interest in light of stressful military deployments currently in Iraq and Afghanistan [7-9, 11, $25,37,38]$. This is the first study to prospectively document, in a large population-based military cohort, newly reported lupus and RA in the context of deployment while differentiating stresses of combat experiences. Compared with nondeployers, deployment with and without combat exposures was not found to be a significant risk factor for newly reported lupus. However, deployment with and without combat exposures was significantly associated with a decreased risk of newly reported RA compared with nondeployers. To better assess the full effects associated with stressful exposures during deployment, deployers with combat exposures were compared with deployers without combat exposures. Compared with noncombat deployers, those with combat exposures had an increased risk of newly reported $\mathrm{RA}$ though not significant $(\mathrm{AOR}=1.24,95 \% \mathrm{CI}$ : 0.96-1.61). This may indicate a stress-induced pathway that elevates the risk for RA among deployers who experience combat $[12,39,40]$. Though long-term follow-up of the Cohort will yield further insight into deployment-related effects, these results are reassuring that compared with nondeployers, there is no apparent increase in risk for newly reported lupus or RA in relation to deployment in support of Iraq and Afghanistan.

The cumulative incidence of lupus reported in this study was elevated compared with previous reports for the US 
TABLE 1: Baseline* characteristics of Millennium Cohort participants with newly reported lupus and rheumatoid arthritis (2001-2008).

\begin{tabular}{|c|c|c|c|c|c|c|c|c|}
\hline \multirow{4}{*}{ Characteristics } & \multicolumn{4}{|c|}{ Lupus } & \multicolumn{4}{|c|}{ Rheumatoid arthritis } \\
\hline & \multirow{2}{*}{\multicolumn{2}{|c|}{$\begin{array}{l}\text { Disease } \\
n=115\end{array}$}} & \multirow{2}{*}{\multicolumn{2}{|c|}{$\begin{array}{l}\text { No disease } \\
n=72,576\end{array}$}} & \multirow{2}{*}{\multicolumn{2}{|c|}{$\begin{array}{c}\text { Disease } \\
n=1,100\end{array}$}} & \multirow{2}{*}{\multicolumn{2}{|c|}{$\begin{array}{l}\text { No disease } \\
n=69,924\end{array}$}} \\
\hline & & & & & & & & \\
\hline & $n$ & $\%^{\dagger}$ & $n$ & $\%^{\dagger}$ & $n$ & $\%^{\dagger}$ & $n$ & $\%^{\dagger}$ \\
\hline \multicolumn{9}{|l|}{ Deployment $^{\ddagger}$} \\
\hline Nondeployed & 75 & 65.2 & 39,874 & 54.9 & 744 & 67.6 & 38,111 & 54.5 \\
\hline Deployed without combat & 22 & 19.1 & 17,985 & 24.7 & 174 & 15.8 & 17,594 & 25.2 \\
\hline Deployed with combat ${ }^{\S}$ & 18 & 15.7 & 14,717 & 20.3 & 182 & 16.6 & 14,219 & 20.3 \\
\hline \multicolumn{9}{|l|}{ Sex } \\
\hline Male & 56 & 48.7 & 51,240 & 70.6 & 760 & 69.1 & 49,321 & 70.5 \\
\hline Female & 59 & 51.3 & 21,336 & 29.4 & 340 & 30.9 & 20,603 & 29.5 \\
\hline \multicolumn{9}{|l|}{ Birth year } \\
\hline Before 1960 & 22 & 19.1 & 13,686 & 18.9 & 381 & 34.6 & 12,629 & 18.1 \\
\hline $1960-1969$ & 41 & 35.7 & 24,131 & 33.3 & 470 & 42.7 & 22,996 & 32.9 \\
\hline 1970-1979 & 41 & 35.7 & 24,020 & 33.1 & 197 & 17.9 & 23,645 & 33.8 \\
\hline 1980 and later & 11 & 9.6 & 10,739 & 14.8 & 52 & 4.7 & 10,654 & 15.2 \\
\hline \multicolumn{9}{|l|}{ Race/ethnicity } \\
\hline Non-Hispanic white & 58 & 50.4 & 51,610 & 71.1 & 673 & 61.2 & 49,882 & 71.3 \\
\hline Non-Hispanic black & 32 & 27.8 & 8,723 & 12.0 & 244 & 22.2 & 8,214 & 11.8 \\
\hline Hispanic & 13 & 11.3 & 4,883 & 6.7 & 90 & 8.2 & 4,718 & 6.8 \\
\hline Other & 12 & 10.4 & 7,360 & 10.1 & 93 & 8.5 & 7,110 & 10.2 \\
\hline \multicolumn{9}{|l|}{ Education } \\
\hline High school or less & 67 & 58.3 & 37,153 & 51.2 & 572 & 52.0 & 35,815 & 51.2 \\
\hline Some college & 25 & 21.7 & 15,797 & 21.8 & 297 & 27.0 & 15,061 & 21.5 \\
\hline Bachelor's or higher degree & 23 & 20.0 & 19,626 & 27.0 & 231 & 21.0 & 19,048 & 27.2 \\
\hline \multicolumn{9}{|l|}{ Marital status } \\
\hline Married & 66 & 57.4 & 42,522 & 58.6 & 793 & 72.1 & 40,514 & 57.9 \\
\hline Not married & 49 & 42.6 & 30,054 & 41.4 & 307 & 27.9 & 29,410 & 42.1 \\
\hline \multicolumn{9}{|l|}{ Service component } \\
\hline Active duty & 64 & 55.7 & 40,745 & 56.1 & 640 & 58.2 & 39,235 & 56.1 \\
\hline Reserve/Guard & 51 & 44.4 & 31,831 & 43.9 & 460 & 41.8 & 30,689 & 43.9 \\
\hline \multicolumn{9}{|l|}{ Military pay grade } \\
\hline Enlisted & 97 & 84.4 & 55,262 & 76.1 & 935 & 85.0 & 53,068 & 75.9 \\
\hline Officer & 18 & 15.7 & 17,314 & 23.9 & 165 & 15.0 & 16,856 & 24.1 \\
\hline \multicolumn{9}{|l|}{ Service branch } \\
\hline Army & 63 & 54.8 & 33,856 & 46.7 & 619 & 56.3 & 32,349 & 46.3 \\
\hline Air Force & 30 & 26.1 & 21,557 & 29.7 & 272 & 24.7 & 20,924 & 29.9 \\
\hline Marine Corps & 4 & 3.5 & 3,880 & 5.4 & 43 & 3.9 & 3,765 & 5.4 \\
\hline Navy/Coast Guard & 18 & 15.7 & 13,283 & 18.3 & 166 & 15.1 & 12,886 & 18.4 \\
\hline \multicolumn{9}{|l|}{ Occupation } \\
\hline Combat specialist & 16 & 13.9 & 14,058 & 19.4 & 185 & 16.8 & 13,588 & 19.4 \\
\hline Health care specialist & 23 & 20.0 & 8,259 & 11.4 & 90 & 8.2 & 8,054 & 11.5 \\
\hline Functional support & 33 & 28.7 & 14,085 & 19.4 & 291 & 26.5 & 13,428 & 19.2 \\
\hline Electrical/mechanical & 12 & 10.4 & 10,156 & 14.0 & 155 & 14.1 & 9,744 & 13.9 \\
\hline Service support & 10 & 8.7 & 6,433 & 8.9 & 125 & 11.4 & 6,139 & 8.8 \\
\hline Other & 21 & 18.3 & 19,585 & 27.0 & 254 & 23.1 & 18,971 & 27.1 \\
\hline \multicolumn{9}{|l|}{ Body mass index $\left(\mathrm{kg} / \mathrm{m}^{2}\right)$} \\
\hline Normal/underweight $(<25)$ & 56 & 48.7 & 29,520 & 40.7 & 320 & 29.1 & 28,790 & 41.2 \\
\hline Overweight $(25$ to <30) & 45 & 39.1 & 35,874 & 49.4 & 604 & 54.9 & 34,441 & 49.3 \\
\hline Obese $(\geq 30)$ & 14 & 12.2 & 7,182 & 9.9 & 176 & 16.0 & 6,693 & 9.6 \\
\hline Smoking status & & & & & & & & \\
\hline Nonsmoker & 68 & 59.1 & 42,572 & 58.7 & 552 & 50.1 & 41,285 & 59.0 \\
\hline Past smoker & 21 & 18.3 & 17,425 & 24.0 & 321 & 29.2 & 16,613 & 23.8 \\
\hline Current smoker & 26 & 22.6 & 12,579 & 17.3 & 228 & 20.7 & 12,026 & 17.2 \\
\hline
\end{tabular}


TABle 1: Continued.

\begin{tabular}{|c|c|c|c|c|c|c|c|c|}
\hline \multirow{4}{*}{ Characteristics } & \multicolumn{4}{|c|}{ Lupus } & \multicolumn{4}{|c|}{ Rheumatoid arthritis } \\
\hline & \multirow{2}{*}{\multicolumn{2}{|c|}{$\begin{array}{l}\text { Disease } \\
n=115\end{array}$}} & \multirow{2}{*}{\multicolumn{2}{|c|}{$\begin{array}{l}\text { No disease } \\
n=72,576\end{array}$}} & \multirow{2}{*}{\multicolumn{2}{|c|}{$\begin{array}{c}\text { Disease } \\
n=1,100\end{array}$}} & \multirow{2}{*}{\multicolumn{2}{|c|}{$\begin{array}{l}\text { No disease } \\
n=69,924\end{array}$}} \\
\hline & & & & & & & & \\
\hline & $n$ & $\%^{\dagger}$ & $n$ & $\%^{\dagger}$ & $n$ & $\%^{\dagger}$ & $n$ & $\%^{\dagger}$ \\
\hline \multicolumn{9}{|l|}{ Alcohol misuse } \\
\hline No & 91 & 79.1 & 58,901 & 81.2 & 894 & 81.3 & 56,766 & 81.2 \\
\hline Yes & 24 & 20.9 & 13,675 & 18.8 & 206 & 18.7 & 13,158 & 18.8 \\
\hline \multicolumn{9}{|l|}{ Mental component score } \\
\hline$>75$ th percentile & 26 & 22.6 & 20,760 & 28.6 & 299 & 27.2 & 19,974 & 28.6 \\
\hline$>50$ th to 75 th percentile & 27 & 23.5 & 17,759 & 24.5 & 248 & 22.6 & 17,166 & 24.6 \\
\hline$>25$ th to 50 th percentile & 23 & 20.0 & 16,266 & 22.4 & 182 & 16.6 & 15,721 & 22.5 \\
\hline 0 to 25 th percentile & 39 & 33.9 & 17,791 & 24.5 & 371 & 33.7 & 17,063 & 24.4 \\
\hline \multicolumn{9}{|l|}{ Physical component score } \\
\hline$>75$ th percentile & 20 & 17.4 & 20,307 & 28.0 & 124 & 11.3 & 19,756 & 28.3 \\
\hline$>50$ th to 75 th percentile & 26 & 22.6 & 18,245 & 25.1 & 168 & 15.3 & 17,664 & 25.3 \\
\hline$>25$ th to 50 th percentile & 22 & 19.1 & 17,135 & 23.6 & 256 & 23.3 & 16,552 & 23.7 \\
\hline 0 to 25 th percentile & 47 & 40.9 & 16,889 & 23.3 & 552 & 50.2 & 15,952 & 22.8 \\
\hline \multicolumn{9}{|c|}{ Chemical and/or biological warfare agents } \\
\hline No & 106 & 92.2 & 68,750 & 94.7 & 968 & 88.0 & 66,446 & 95.0 \\
\hline Yes, 1 or more times & 9 & 7.8 & 3,826 & 5.3 & 132 & 12.0 & 3,478 & 5.0 \\
\hline \multicolumn{9}{|c|}{ Hazards requiring personal protective equipment } \\
\hline No & 65 & 56.5 & 31,622 & 43.6 & 476 & 43.3 & 30,558 & 43.7 \\
\hline Yes & 46 & 40.0 & 39,420 & 54.3 & 584 & 53.1 & 37,921 & 54.2 \\
\hline Do not know & 4 & 3.5 & 1,534 & 2.1 & 40 & 3.6 & 1,445 & 2.1 \\
\hline \multicolumn{9}{|c|}{ Routine skin contact with paint, solvents, substances } \\
\hline No & 74 & 64.4 & 50,431 & 69.5 & 669 & 60.8 & 48,912 & 70.0 \\
\hline Yes & 33 & 28.7 & 19,276 & 26.6 & 363 & 33.0 & 2,719 & 3.9 \\
\hline Do not know & 8 & 7.0 & 2,869 & 4.0 & 68 & 6.2 & 2,719 & 3.9 \\
\hline \multicolumn{9}{|c|}{ Microwaves (excluding microwave ovens) } \\
\hline No & 83 & 72.2 & 53,942 & 74.3 & 718 & 65.3 & 52,253 & 74.7 \\
\hline Yes & 24 & 20.9 & 12,818 & 17.7 & 232 & 21.1 & 12,165 & 17.4 \\
\hline Do not know & 8 & 7.0 & 5,816 & 8.0 & 150 & 13.6 & 5,506 & 7.9 \\
\hline \multicolumn{9}{|c|}{ Pesticides (creams, sprays, uniform treatments) } \\
\hline No & 71 & 61.7 & 46,506 & 64.1 & 606 & 55.1 & 45,091 & 64.5 \\
\hline Yes & 35 & 30.4 & 19,652 & 27.1 & 378 & 34.4 & 18,709 & 26.8 \\
\hline Do not know & 9 & 7.8 & 6,418 & 8.8 & 116 & 10.6 & 6,124 & 8.8 \\
\hline \multicolumn{9}{|c|}{ Pesticides (environment, living facilities) } \\
\hline No & 63 & 54.8 & 42.194 & 58.1 & 523 & 47.6 & 40,985 & 58.6 \\
\hline Yes & 37 & 32.2 & 20,508 & 28.3 & 410 & 37.3 & 19,462 & 27.8 \\
\hline Do not know & 15 & 13.0 & 9,874 & 13.6 & 167 & 15.2 & 9,477 & 13.6 \\
\hline
\end{tabular}

* Population excludes participants with missing baseline covariates, may not represent whole study population.

${ }^{\dagger}$ Percentages may not sum to 100 because of rounding.

₹Deployed in support of the operations in Iraq and Afghanistan.

§Self-report of personally witnessing or being exposed to a person's death due to war or disaster, physical abuse, dead and/or decomposing bodies, maimed soldiers or civilians, prisoners of war, or refugees.

"Alcohol misuse is defined as at least 1 positive response to the CAGE questions (i.e., Cutting down, Annoyance by criticism, Guilty feeling, and Eye-openers) $[32,33]$.

population (systemic lupus erythematosus: 0.02 to 0.08 per 1000 person-years) [41-43]. It is difficult to compare these rates, because military personnel may have been exposed to unique environmental and occupational risk factors not typically experienced in the general population. However, misclassification of this disorder may have occurred, because participants reporting lupus may have been diagnosed with any form of lupus and not SLE specifically. This is due to the questionnaire not restricting which type of lupus to be reported. 
TABle 2: Odds of newly reported lupus and military deployment, the Millennium Cohort Study $(N=77,811)$.

\begin{tabular}{|c|c|c|c|c|c|c|}
\hline \multirow{2}{*}{ Characteristics } & \multicolumn{2}{|c|}{ Unadjusted } & \multicolumn{2}{|c|}{ Final model* } & \multicolumn{2}{|c|}{ Confirmed case model $^{\dagger}$} \\
\hline & OR & $95 \% \mathrm{CI}$ & $\mathrm{AOR}^{\ddagger}$ & $95 \% \mathrm{CI}$ & $\mathrm{AOR}^{\ddagger}$ & $95 \% \mathrm{CI}$ \\
\hline \multicolumn{7}{|l|}{ Deployment $^{\S}$} \\
\hline Nondeployed & $1.00^{9}$ & & $1.00^{\circ}$ & & $1.00^{\natural}$ & \\
\hline Deployed without combat & 0.85 & $0.53-1.35$ & 0.91 & $0.55-1.50$ & 0.28 & $0.04-1.82$ \\
\hline Deployed with combat ${ }^{\#}$ & 0.64 & $0.37-1.09$ & 0.66 & $0.37-1.19$ & 0.32 & $0.05-2.11$ \\
\hline \multicolumn{7}{|l|}{ Sex } \\
\hline Male & $1.00^{\circ}$ & & $1.00^{\pi}$ & & $1.00^{\curvearrowleft}$ & \\
\hline Female & 2.42 & $1.69-3.48$ & 1.81 & $1.20-2.72$ & 4.24 & $1.14-15.81$ \\
\hline \multicolumn{7}{|l|}{ Birth year } \\
\hline Before 1960 & $1.00^{\natural}$ & & $1.00^{\circ}$ & & $1.00^{\natural}$ & \\
\hline 1960-1969 & 1.23 & $0.74-2.04$ & 1.26 & $0.75-2.11$ & 0.73 & $0.08-6.67$ \\
\hline $1970-1979$ & 1.36 & $0.82-2.27$ & 1.44 & $0.86-2.44$ & 0.51 & $0.05-5.22$ \\
\hline 1980 and later & 1.23 & $0.60-2.51$ & 1.13 & $0.54-2.37$ & 1.22 & $0.10-15.21$ \\
\hline \multicolumn{7}{|l|}{ Race/ethnicity } \\
\hline Non-Hispanic white & $1.00^{\text {ๆ }}$ & & $1.00^{\natural}$ & & $1.00^{\natural}$ & \\
\hline Non-Hispanic black & 3.24 & $2.12-4.95$ & 2.66 & $1.70-4.17$ & 2.41 & $0.60-9.71$ \\
\hline Hispanic & 2.47 & $1.35-4.54$ & 2.35 & $1.27-4.36$ & 1.15 & $0.13-10.30$ \\
\hline Other & 1.29 & $0.69-2.41$ & 1.38 & $0.74-2.58$ & 0.72 & $0.09-6.01$ \\
\hline \multicolumn{7}{|l|}{ Education } \\
\hline High school or less & $1.00^{\circ}$ & & & & & \\
\hline Some college & 1.41 & $0.93-2.14$ & & & & \\
\hline Bachelor's or higher degree & 0.87 & $0.57-1.31$ & & & & \\
\hline \multicolumn{7}{|l|}{ Marital status } \\
\hline Married & $1.00^{\text {ๆ }}$ & & & & & \\
\hline Not married & 1.67 & $1.18-2.35$ & & & & \\
\hline \multicolumn{7}{|l|}{ Service component } \\
\hline Active duty & $1.00^{\circ}$ & & & & & \\
\hline Reserve/Guard & 0.85 & $0.60-1.21$ & & & & \\
\hline \multicolumn{7}{|l|}{ Military pay grade } \\
\hline Enlisted & $1.00^{\natural}$ & & & & & \\
\hline Officer & 0.58 & $0.37-0.91$ & & & & \\
\hline \multicolumn{7}{|l|}{ Service branch } \\
\hline Army & $1.00^{\natural}$ & & & & & \\
\hline Air Force & 0.69 & $0.47-1.06$ & & & & \\
\hline Marine Corps & 0.80 & $0.32-2.00$ & & & & \\
\hline Navy/Coast Guard & 0.77 & $0.47-1.28$ & & & & \\
\hline \multicolumn{7}{|l|}{ Occupation } \\
\hline Functional support & $1.00^{\natural}$ & & & & & \\
\hline Combat specialist & 0.53 & $0.28-1.00$ & & & & \\
\hline Health care specialist & 1.25 & $0.73-2.13$ & & & & \\
\hline Electrical/mechanical & 0.73 & $0.40-1.35$ & & & & \\
\hline Service support & 0.71 & $0.37-1.35$ & & & & \\
\hline Other & 0.67 & $0.40-1.11$ & & & & \\
\hline \multicolumn{7}{|l|}{ Body mass index $\left(\mathrm{kg} / \mathrm{m}^{2}\right)$} \\
\hline Normal/underweight $(<25)$ & $1.00^{\natural}$ & & & & & \\
\hline Overweight $(25$ to $<30)$ & 0.82 & $0.56-1.20$ & & & & \\
\hline Obese $(\geq 30)$ & 1.44 & $0.93-2.24$ & & & & \\
\hline \multicolumn{7}{|l|}{ Smoking status } \\
\hline Nonsmoker & $1.00^{\circ}$ & & & & & \\
\hline Past smoker & 1.04 & $0.69-1.57$ & & & & \\
\hline Current smoker & 1.27 & $0.77-2.09$ & & & & \\
\hline
\end{tabular}


TABle 2: Continued.

\begin{tabular}{|c|c|c|c|c|c|c|}
\hline \multirow{2}{*}{ Characteristics } & \multicolumn{2}{|c|}{ Unadjusted } & \multicolumn{2}{|c|}{ Final model* } & \multicolumn{2}{|c|}{ Confirmed case model ${ }^{\dagger}$} \\
\hline & OR & $95 \% \mathrm{CI}$ & $\mathrm{AOR}^{\ddagger}$ & $95 \% \mathrm{CI}$ & $\mathrm{AOR}^{\ddagger}$ & $95 \% \mathrm{CI}$ \\
\hline \multicolumn{7}{|l|}{ Alcohol misuse ${ }^{* *}$} \\
\hline No & $1.00^{\circ}$ & & & & & \\
\hline Yes & 1.13 & $0.75-1.71$ & & & & \\
\hline \multicolumn{7}{|l|}{ Mental component score } \\
\hline$>75$ th percentile & $1.00^{\circ}$ & & $1.00^{\circ}$ & & & \\
\hline$>50$ th to 75 th percentile & 1.16 & $0.72-1.85$ & 1.25 & $0.75-2.07$ & & \\
\hline$>25$ th to 50 th percentile & 0.84 & $0.50-1.42$ & 0.89 & $0.52-1.53$ & & \\
\hline 0 to 25 th percentile & 1.96 & $1.26-3.05$ & 1.68 & $1.06-2.66$ & & \\
\hline \multicolumn{7}{|l|}{ Physical component score } \\
\hline$>75$ th percentile & $1.00^{\circ}$ & & $1.00^{\circ}$ & & & \\
\hline$>50$ th to 75 th percentile & 0.97 & $0.51-1.84$ & 1.08 & $0.55-2.14$ & & \\
\hline$>25$ th to 50 th percentile & 1.73 & $0.95-3.14$ & 1.79 & $0.96-3.34$ & & \\
\hline 0 to 25 th percentile & 4.27 & $2.50-7.29$ & 4.34 & $2.51-7.49$ & & \\
\hline \multicolumn{7}{|c|}{ Chemical/biological warfare agents } \\
\hline No & $1.00^{\circ}$ & & & & & \\
\hline Yes, 1 more times & 0.68 & $0.45-1.02$ & & & & \\
\hline \multicolumn{7}{|c|}{ Hazards requiring personal protective equipment } \\
\hline No & $1.00^{\pi}$ & & & & & \\
\hline Yes & 0.70 & $0.49-1.01$ & & & & \\
\hline Do not know & 1.21 & $0.43-3.38$ & & & & \\
\hline \multicolumn{7}{|c|}{ Routine skin contact with paint, solvents, substances } \\
\hline No & $1.00^{\uparrow}$ & & & & & \\
\hline Yes & 1.18 & $0.81-1.73$ & & & & \\
\hline Do not know & 2.20 & $1.12-4.32$ & & & & \\
\hline \multicolumn{7}{|c|}{ Microwaves (excluding microwave ovens) } \\
\hline No & $1.00^{\natural}$ & & & & & \\
\hline Yes & 1.07 & $0.69-1.67$ & & & & \\
\hline Do not know & 1.40 & $0.82-2.42$ & & & & \\
\hline \multicolumn{7}{|c|}{ Pesticides (creams, sprays, uniform treatments) } \\
\hline No & $1.00^{\circ}$ & & & & & \\
\hline Yes & 1.17 & $0.81-1.69$ & & & & \\
\hline Do not know & 0.87 & $0.44-1.73$ & & & & \\
\hline \multicolumn{7}{|c|}{ Pesticides (environment, living facilities) } \\
\hline No & $1.00^{\natural}$ & & & & & \\
\hline Yes & 1.15 & $0.79-1.66$ & & & & \\
\hline Do not know & 1.02 & $0.58-1.78$ & & & & \\
\hline Time (years) & 1.42 & $1.31-1.55$ & 1.38 & $1.26-1.50$ & 1.42 & $1.08-1.88$ \\
\hline
\end{tabular}

AOR, adjusted odds ratio; $\mathrm{CI}$, confidence interval; OR, odds ratio.

* Final model established by removing nonsignificant, nonconfounding covariates from the full model.

${ }^{\dagger}$ Confirmed cases model is among active-duty, nonseparated participants. There were not enough confirmed cases for mental and physical component scores to be tested in the model.

¥Adjusted odds ratios and associated 95\% confidence intervals are adjusted for all other variables in the table.

$\S$ Deployed in support of the operations in Iraq and Afghanistan.

"Indicates reference category.

\#Self-report of personally witnessing or being exposed to a person's death due to war or disaster, physical abuse, dead and/or decomposing bodies, maimed soldiers or civilians, prisoners of war, or refugees.

**Alcohol misuse is defined as at least 1 positive response to the CAGE questions (i.e., cutting down, annoyance by criticism, guilty feeling, and eye-openers) $[32,33]$. 
TABle 3: Odds of newly reported rheumatoid arthritis and military deployment, the Millennium Cohort Study $(N=76,003)$.

\begin{tabular}{|c|c|c|c|c|c|c|}
\hline \multirow{2}{*}{ Characteristics } & \multicolumn{2}{|c|}{ Unadjusted } & \multicolumn{2}{|c|}{ Final model* } & \multicolumn{2}{|c|}{ Confirmed case model $^{\dagger}$} \\
\hline & OR & $95 \% \mathrm{CI}$ & $\mathrm{AOR}^{\ddagger}$ & $95 \% \mathrm{CI}$ & $\mathrm{AOR}^{\ddagger}$ & $95 \% \mathrm{CI}$ \\
\hline \multicolumn{7}{|l|}{ Deployment ${ }^{\S}$} \\
\hline Nondeployed & $1.00^{\Uparrow}$ & & $1.00^{\circ}$ & & $1.00^{\circ}$ & \\
\hline Deployed without combat & 0.47 & $0.39-0.56$ & 0.59 & $0.48-0.73$ & 0.70 & $0.33-1.49$ \\
\hline Deployed with combat ${ }^{\#}$ & 0.69 & $0.59-0.81$ & 0.74 & $0.62-0.88$ & 0.83 & $0.38-1.77$ \\
\hline \multicolumn{7}{|l|}{ Sex } \\
\hline Male & $1.00^{\circ}$ & & $1.00^{\circ}$ & & $1.00^{\circ}$ & \\
\hline Female & 1.16 & $1.02-1.31$ & 1.26 & $1.08-1.47$ & 1.96 & $0.88-4.37$ \\
\hline \multicolumn{7}{|l|}{ Birth year } \\
\hline Before 1960 & $1.00^{\natural}$ & & $1.00^{\natural}$ & & $1.00^{\circ}$ & \\
\hline $1960-1969$ & 0.72 & $0.63-0.82$ & 0.64 & $0.55-0.74$ & 0.94 & $0.30-2.95$ \\
\hline 1970-1979 & 0.33 & $0.27-0.39$ & 0.31 & $0.26-0.38$ & 0.32 & $0.09-1.20$ \\
\hline 1980 and later & 0.29 & $0.22-0.39$ & 0.26 & $0.19-0.35$ & 0.37 & $0.08-1.78$ \\
\hline \multicolumn{7}{|l|}{ Race/ethnicity } \\
\hline Non-Hispanic white & $1.00^{\natural}$ & & $1.00^{\natural}$ & & $1.00^{\natural}$ & \\
\hline Non-Hispanic black & 2.27 & $1.97-2.62$ & 1.77 & $1.51-2.08$ & 0.58 & $0.21-1.60$ \\
\hline Hispanic & 1.49 & $1.20-1.85$ & 1.40 & $1.11-1.76$ & 1.17 & $0.41-3.36$ \\
\hline Other & 0.92 & $0.74-1.13$ & 1.00 & $0.79-1.27$ & 1.26 & $0.42-3.78$ \\
\hline \multicolumn{7}{|l|}{ Education } \\
\hline High school or less & $1.00^{\natural}$ & & $1.00^{\text {ๆ }}$ & & $1.00^{\circ}$ & \\
\hline Some college & 1.67 & $1.47-1.91$ & 1.21 & $1.05-1.39$ & 1.91 & $0.92-3.94$ \\
\hline Bachelor's or higher degree & 0.88 & $0.76-1.01$ & 1.10 & $0.93-1.30$ & 1.57 & $0.64-3.88$ \\
\hline \multicolumn{7}{|l|}{ Marital status } \\
\hline Married & $1.00^{\natural}$ & & & & & \\
\hline Not married & 0.98 & $0.87-1.11$ & & & & \\
\hline \multicolumn{7}{|l|}{ Service component } \\
\hline Active duty & $1.00^{\circ}$ & & $1.00^{\natural}$ & & $1.00^{\circ}$ & \\
\hline Reserve/Guard & 0.83 & $0.74-0.93$ & 0.73 & $0.64-0.83$ & 0.14 & $0.02-1.35$ \\
\hline \multicolumn{7}{|l|}{ Military pay grade } \\
\hline Enlisted & $1.00^{\curvearrowleft}$ & & $1.00^{\circ}$ & & $1.00^{\circ}$ & \\
\hline Officer & 0.45 & $0.39-0.53$ & 0.60 & $0.48-0.74$ & 0.17 & $0.05-0.61$ \\
\hline \multicolumn{7}{|l|}{ Service branch } \\
\hline Army & $1.00^{\Uparrow}$ & & $1.00^{\circ}$ & & & \\
\hline Air Force & 0.63 & $0.55-0.73$ & 0.79 & $0.67-0.92$ & & \\
\hline Marine Corps & 0.71 & $0.53-0.96$ & 1.01 & $0.74-1.37$ & & \\
\hline Navy/Coast Guard & 0.67 & $0.57-0.79$ & 0.74 & $0.62-0.89$ & & \\
\hline \multicolumn{7}{|l|}{ Occupation } \\
\hline Functional support & $1.00^{\natural}$ & & $1.00^{\natural}$ & & $1.00^{\circ}$ & \\
\hline Combat specialist & 0.80 & $0.66-0.96$ & 1.09 & $0.90-1.32$ & 0.78 & $0.26-2.35$ \\
\hline Health care specialist & 0.60 & $0.48-0.76$ & 0.66 & $0.52-0.85$ & 0.50 & $0.16-1.55$ \\
\hline Electrical/mechanical & 1.03 & $0.85-1.24$ & 1.07 & $0.87-1.36$ & 1.12 & $0.46-2.74$ \\
\hline Service support & 1.19 & $0.97-1.44$ & 1.11 & $0.91-1.36$ & 1.52 & $0.56-4.12$ \\
\hline Other & 0.87 & $0.74-1.02$ & 1.00 & $0.85-1.19$ & 0.40 & $0.16-0.97$ \\
\hline \multicolumn{7}{|l|}{ Body mass index $\left(\mathrm{kg} / \mathrm{m}^{2}\right)$} \\
\hline Normal/Underweight $(<25)$ & $1.00^{\circ}$ & & $1.00^{\circ}$ & & $1.00^{\natural}$ & \\
\hline Overweight $(25$ to <30) & 1.34 & $1.16-1.54$ & 1.11 & $0.95-1.29$ & 0.77 & $0.38-1.56$ \\
\hline Obese $(\geq 30)$ & 2.41 & $2.07-2.80$ & 1.41 & $1.20-1.67$ & 1.20 & $0.49-2.91$ \\
\hline \multicolumn{7}{|l|}{ Smoking status } \\
\hline Nonsmoker & $1.00^{\curvearrowleft}$ & & $1.00^{\circ}$ & & $1.00^{\circ}$ & \\
\hline Past smoker & 1.39 & $1.22-1.58$ & 1.13 & $0.99-1.30$ & 1.63 & $0.84-3.14$ \\
\hline Current smoker & 1.54 & $1.33-1.80$ & 1.19 & $1.02-1.40$ & 0.56 & $0.20-1.56$ \\
\hline
\end{tabular}


TABle 3: Continued.

\begin{tabular}{|c|c|c|c|c|c|c|}
\hline \multirow{2}{*}{ Characteristics } & \multicolumn{2}{|c|}{ Unadjusted } & \multicolumn{2}{|c|}{ Final model* } & \multicolumn{2}{|c|}{ 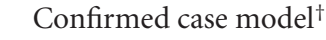 } \\
\hline & OR & $95 \% \mathrm{CI}$ & $\mathrm{AOR}^{\ddagger}$ & $95 \% \mathrm{CI}$ & $\mathrm{AOR}^{\ddagger}$ & $95 \% \mathrm{CI}$ \\
\hline \multicolumn{7}{|l|}{ Alcohol misuse ${ }^{* *}$} \\
\hline No & $1.00^{\natural}$ & & & & & \\
\hline Yes & 1.18 & $1.04-1.36$ & & & & \\
\hline \multicolumn{7}{|l|}{ Mental component score } \\
\hline$>75$ th percentile & $1.00^{\circ}$ & & $1.00^{\circ}$ & & $1.00^{\circ}$ & \\
\hline$>50$ th to 75 th percentile & 0.73 & $0.62-0.85$ & 0.89 & $0.75-1.06$ & 0.72 & $0.35-1.48$ \\
\hline$>25$ th to 50 th percentile & 0.87 & $0.75-1.02$ & 1.04 & $0.88-1.23$ & 0.73 & $0.36-1.49$ \\
\hline 0 to 25 th percentile & 1.67 & $1.46-1.92$ & 1.64 & $1.42-1.91$ & 0.85 & $0.42-1.70$ \\
\hline \multicolumn{7}{|l|}{ Physical component score } \\
\hline$>75$ th percentile & $1.00^{\circ}$ & & $1.00^{\circ}$ & & $1.00^{\circ}$ & \\
\hline$>50$ th to 75 th percentile & 1.40 & $1.05-1.86$ & 1.38 & $1.02-1.88$ & 1.34 & $0.37-4.83$ \\
\hline$>25$ th to 50 th percentile & 2.99 & $2.30-3.90$ & 2.72 & $2.06-3.61$ & 2.56 & $0.78-8.35$ \\
\hline 0 to 25 th percentile & 12.25 & $9.60-15.63$ & 9.03 & $6.95-11.74$ & 7.89 & $2.48-25.10$ \\
\hline \multicolumn{7}{|c|}{ Chemical/biological warfare agents } \\
\hline No & $1.00^{\circ}$ & & $1.00^{\circ}$ & & $1.00^{\circ}$ & \\
\hline Yes, 1 or more times & 0.98 & $0.85-1.12$ & 1.47 & $1.22-1.78$ & 1.43 & $0.49-4.18$ \\
\hline \multicolumn{7}{|c|}{ Hazards requiring personal protective equipment } \\
\hline No & $1.00^{\natural}$ & & & & & \\
\hline Yes & 0.96 & $0.85-1.07$ & & & & \\
\hline Do not know & 1.59 & $1.17-2.18$ & & & & \\
\hline \multicolumn{7}{|c|}{ Routine skin contact with paint, solvents, substances } \\
\hline No & $1.00^{\natural}$ & & & & & \\
\hline Yes & 1.39 & $1.24-1.56$ & & & & \\
\hline Do not know & 1.74 & $1.38-2.19$ & & & & \\
\hline \multicolumn{7}{|c|}{ Microwaves (excluding microwave ovens) } \\
\hline No & $1.00^{\circ}$ & & $1.00^{\natural}$ & & $1.00^{\circ}$ & \\
\hline Yes & 1.44 & $1.26-1.64$ & 1.14 & $0.99-1.31$ & 0.80 & $0.36-1.78$ \\
\hline Do not know & 1.63 & $1.38-1.92$ & 1.25 & $1.05-1.50$ & 1.58 & $0.60-4.16$ \\
\hline \multicolumn{7}{|c|}{ Pesticides (creams, sprays, uniform treatments) } \\
\hline No & $1.00^{\natural}$ & & & & & \\
\hline Yes & 1.39 & $1.24-1.57$ & & & & \\
\hline Do not know & 1.36 & $1.13-1.64$ & & & & \\
\hline \multicolumn{7}{|c|}{ Pesticides (environment, living facilities) } \\
\hline No & $1.00^{\natural}$ & & & & & \\
\hline Yes & 1.47 & $1.30-1.65$ & & & & \\
\hline Do not know & 1.22 & $1.04-1.45$ & & & & \\
\hline Time (years) & 1.44 & $1.40-1.48$ & 1.31 & $1.27-1.35$ & 1.19 & $0.99-1.44$ \\
\hline
\end{tabular}

$\mathrm{AOR}$, adjusted odds ratio; $\mathrm{CI}$, confidence interval; $\mathrm{OR}$, odds ratio.

* Final model established by removing nonsignificant, nonconfounding covariates from the full model.

${ }^{\dagger}$ Confirmed cases model is among active-duty, nonseparated participants. There were not enough confirmed cases for service branch to be tested in the model.

${ }^{\ddagger}$ Adjusted odds ratios and associated $95 \%$ confidence intervals are adjusted for all other variables in the table.

$\S$ Deployed in support of the operations in Iraq and Afghanistan.

"Indicates reference category.

\#Self-report of personally witnessing or being exposed to a person's death due to war or disaster, physical abuse, dead and/or decomposing bodies, maimed soldiers or civilians, prisoners of war, or refugees.

** Alcohol misuse is defined as at least 1 positive response to the CAGE questions (i.e., cutting down, annoyance by criticism, guilty feeling, and eye-openers) $[32,33]$.

Previous literature has documented that environmental and occupational exposures were associated with an increased risk of lupus $[16,18-20]$; however, these predictors were not strong risk factors for lupus development in this study and were not included in the final model. Though incidence rates have been reported to be much higher for women, and non-Hispanic black and Hispanic participants in the US population $[3,41,43,44]$, the incidence of 
lupus has not been thoroughly investigated in a military population, making an appropriate comparison difficult. Despite these differences, lower mental and physical health was associated with increased risk of lupus, which was consistent with previous research in the US population [45].

Similar to lupus, there was a higher cumulative incidence of self-reported RA in this study compared with previous results in the US population ( 0.24 to 0.68 per 1000 personyears) $[1,5,42]$. This may be a result of those with any form of arthritis endorsing RA, as the questionnaire did not provide a definition of RA. Also, the incidence rates for women and non-Hispanic black and Hispanic participants have been reported higher for the US population than in the current study $[1,5]$. These findings, however, were consistent with previous reports of greater RA risk associated with lower mental and physical health status $[45,46]$, higher body mass index $[1,47,48]$, smoking $[1,47,48]$, and less education $[1,47]$. Additionally, the documented average age of newonset RA is between 30 and 55 years of age among the general population [1], and the current study consistently found a lower risk associated with younger age.

To our knowledge, an increased risk of RA among those self-reporting exposure to chemical or biological warfare agents has not been reported. Due to the lack of evidence of chemical or biological warfare agents existing in the current operations in Iraq and Afghanistan, this association may be a self-perceived exposure or the result of overreporting bias. In addition, the association between RA and exposure to microwaves (excluding microwave ovens) has not been documented. While in vivo and in vitro studies suggest low exposures to microwaves may cause changes in the immune system, and neurological and behavioral effects, the true biological responses due to microwave exposures are still under investigation [49].

This study had several limitations. The current analyses used self-reported data from the Millennium Cohort questionnaire; however, previous studies of this Cohort suggest it is representative of US military personnel, the data reported by its participants are reliable and were unaffected by the participant's health status prior to enrollment $[22,23,36,50$ 57]. Also, the questionnaire did not specify which type of lupus or define RA which may have resulted in misclassification of both outcomes. With a large sample size and population-based design, conducting clinical examinations to confirm self-reported diagnoses was not feasible. The documented validity of self-reported provider-diagnosed data varies widely for lupus $(21 \%-84 \%)$ and RA $(7 \%-96 \%)$ [58-63]. Therefore, an electronic medical records review was performed for both outcomes using ICD-9 codes in Armed Forces Health Longitudinal Technology Application and Military Health System databases. This review could only be conducted for active-duty members diagnosed while in service. Confirmation of 13 out of 33 (39.4\%) lupus and 39 out of 281 (13.9\%) RA self-reported diagnoses were found. Thus, there may be substantial misclassification of both outcomes. The sensitivity analyses among the confirmed diagnoses resulted in stability of the measures of association between deployment and lupus and RA (see "Confirmed case models", Tables 2 and 3 ).

Another important limitation was lupus and RA are rare outcomes resulting in few newly reported cases occurring during the average 5.6 years of follow-up. This may ultimately affect the precision of the estimates that will only be lessened with additional follow-up and subsequent cases. Also, deployment included those deployed for 1 or more days, so those on shorter deployments had fewer days exposed than those on longer deployments. Furthermore, deployed participants that did not affirmatively respond to the questions defining combat exposure may have been exposed to combat situations that were not addressed in this study including receiving small arms fire, or being attacked or ambushed. Lastly, the Millennium Cohort Study does not obtain information on diet, family history, genetic risk factors, or medication, making it difficult to thoroughly examine these exposures as possible risk factors for these diseases.

Despite these limitations, this study has several strengths. This is the first study to prospectively investigate whether military deployment status is associated with newly reported provider-diagnosis of lupus and RA, while also being able to evaluate specific military exposures and adjust for negative health behaviors such as alcohol misuse and tobacco use. Additionally, the prospective design of the Cohort allowed for assessment at baseline and follow-up of the same individuals accounting for time-varying covariates. Lastly, a large sample size, representing both men and women, all branches of the military, active duty, and Reserve and National Guard personnel during and after service, enhanced statistical power for assessing these chronic diseases while considering multiple potential confounders.

In conclusion, newly reported lupus was not associated with military deployment in support of the current operations in Iraq and Afghanistan when compared with nondeployers. These findings, however, suggest a significantly decreased risk of newly reported RA for deployers with and without combat exposures and may be due to a selection effect for deployment. Demographic and general health characteristics and specific exposures were also associated with lupus and RA development in this population. While this study did not find deployment to be a significant risk factor for development of lupus or RA, it is important to further investigate these associations in the future, as the incidence of lupus and RA may progress over time. This study highlights the strengths of prospectively addressing long-term health concerns associated with occupational and environmental exposures among military personnel.

\section{Acknowledgments}

This work represents report 10-36, supported by the US Department of Defense, under work unit no. 60002. The views expressed in this article are those of the authors and do not reflect the official policy or position of the Department of the Navy, the Department of the Army, the Department of the Air Force, the Department of Defense, the Department of Veterans Affairs, or the US Government. 
Approved for public release; distribution is unlimited. This research was conducted in compliance with all applicable federal regulations governing the protection of human subjects in research (protocol NHRC.2000.0007). The authors are indebted to the Millennium Cohort Study participants, without whom these analyses would not be possible. We thank Scott Seggerman from the Defense Manpower Data Center, Monterey, Calif, USA, and the professionals from the US Army Medical Research and Materiel Command, especially those from the Military Operational Medicine Research Program, Fort Detrick, Md, USA. In addition to the authors, the Millennium Cohort Study Team includes Paul Amoroso, Gregory Gray, Tomoko Hooper, James Riddle, and Timothy Wells. We thank Melissa Bagnell, Gina Creaven, James Davies, Gia Gumbs, Jaime Horton, Isabel Jacobson, Cynthia LeardMann, Travis Leleu, Gordon Lynch, Jamie McGrew, Hope McMaster, Amanda Pietrucha, Teresa Powell, Donald Sandweiss, Kari Sausedo, Amber Seelig, Beverly Sheppard, Katherine Snell, Steven Spiegle, Marleen Welsh, Martin White, James Whitmer and Charlene Wong from the Deployment Health Research Department and Michelle Stoia from the Naval Health Research Center, San Diego, Calif, USA. The authors appreciate the support of the Henry M. Jackson Foundation for the Advancement of Military Medicine, Rockville, Md, USA. VA Puget Sound provided support for Dr. Boyko's participation in this research.

\section{References}

[1] Y. Alamanos and A. A. Drosos, "Epidemiology of adult rheumatoid arthritis," Autoimmunity Reviews, vol. 4, no. 3, pp. 130-136, 2005.

[2] G. S. Cooper, M. L. K. Bynum, and E. C. Somers, "Recent insights in the epidemiology of autoimmune diseases: improved prevalence estimates and understanding of clustering of diseases," Journal of Autoimmunity, vol. 33, no. 3-4, pp. 197-207, 2009.

[3] D. Fairweather, S. Frisancho-Kiss, and N. R. Rose, "Sex differences in autoimmune disease from a pathological perspective," American Journal of Pathology, vol. 173, no. 3, pp. 600-609, 2008.

[4] C. G. Helmick, D. T. Felson, R. C. Lawrence et al., "Estimates of the prevalence of arthritis and other rheumatic conditions in the United States: part I," Arthritis and Rheumatism, vol. 58, no. 1, pp. 15-25, 2008.

[5] D. L. Jacobson, S. J. Gange, N. R. Rose, and N. M. H. Graham, "Epidemiology and estimated population burden of selected autoimmune diseases in the United States," Clinical Immunology and Immunopathology, vol. 84, no. 3, pp. 223243, 1997.

[6] The Autoimmune Diseases Coordinating Committee, Progress in Autoimmune Diseases Research: Report to Congress, U.S. Department of Health and Human Services, 2005.

[7] N. S. Granado, T. C. Smith, G. M. Swanson et al., "Newly reported hypertension after military combat deployment in a large population-based study," Hypertension, vol. 54, no. 5, pp. 966-973, 2009.

[8] I. G. Jacobson, M. A. K. Ryan, T. I. Hooper et al., "Alcohol use and alcohol-related problems before and after military combat deployment," Journal of the American Medical Association, vol. 300, no. 6, pp. 663-675, 2008.
[9] B. Smith, M. A. K. Ryan, D. L. Wingard, T. L. Patterson, D. J. Slymen, and C. A. Macera, "Cigarette smoking and military deployment: a prospective evaluation," American Journal of Preventive Medicine, vol. 35, no. 6, pp. 539-546, 2008.

[10] B. Smith, C. A. Wong, T. C. Smith, E. J. Boyko, G. D. Gackstetter, and M. A. K. Ryan, "Newly reported respiratory symptoms and conditions among military personnel deployed to iraq and afghanistan: a prospective population-based study," American Journal of Epidemiology, vol. 170, no. 11, pp. 1433-1442, 2009.

[11] T. C. Smith, M. A. K. Ryan, D. L. Wingard, D. J. Slymen, J. F. Sallis, and D. Kritz-Silverstein, "New onset and persistent symptoms of post-traumatic stress disorder self reported after deployment and combat exposures: prospective population based US military cohort study," British Medical Journal, vol. 336, no. 7640, pp. 366-371, 2008.

[12] J. A. Boscarino, "Posttraumatic stress disorder and physical illness: results from clinical and epidemiologic studies," Annals of the New York Academy of Sciences, vol. 1032, pp. 141-153, 2004.

[13] E. P. Grady, M. T. Carpenter, C. D. Koenig, S. A. Older, and D. F. Battafarano, "Rheumatic findings in Gulf War veterans," Archives of Internal Medicine, vol. 158, no. 4, pp. 367-371, 1998.

[14] T. C. Smith, G. C. Gray, and J. D. Knoke, "Is systemic lupus erythematosus, amyotrophic lateral sclerosis, or fibromyalgia associated with Persian Gulf War service? An examination of Department of Defense hospitalization data," American Journal of Epidemiology, vol. 151, no. 11, pp. 1053-1059, 2000.

[15] A. R. Olsson, T. Skogh, O. Axelson, and G. Wingren, "Occupations and exposures in the work environment as determinants for rheumatoid arthritis," Occupational and Environmental Medicine, vol. 61, no. 3, pp. 233-238, 2004.

[16] C. G. Parks and G. S. Cooper, "Occupational exposures and risk of systemic lupus erythematosus: a review of the evidence and exposure assessment methods in population-and clinicbased studies," Lupus, vol. 15, no. 11, pp. 728-736, 2006.

[17] P. Sarzi-Puttini, F. Atzeni, L. Iaccarino, and A. Doria, "Environment and systemic lupus erythematosus: an overview," Autoimmunity, vol. 38, no. 7, pp. 465-472, 2005.

[18] M. P. Holsapple, "Autoimmunity by pesticides: a critical review of the state of the science," Toxicology Letters, vol. 127, no. 1-3, pp. 101-109, 2002.

[19] E. V. Hess, "Environmental chemicals and autoimmune disease: cause and effect," Toxicology, vol. 181-182, pp. 65-70, 2002.

[20] D. D'Cruz, “Autoimmune diseases associated with drugs, chemicals and environmental factors," Toxicology Letters, vol. 112-113, pp. 421-432, 2000.

[21] N. Kaminski, K. Faubert, and M. P. Holsapple, "Toxic responses of the immune system," in Casarett \& Doull's Toxicology The Basic Science of Poisons, C. Klaassen, Ed., chapter 12, pp. 485-555, McGraw-Hill Health Professions Division, New York, NY, USA, 5th edition, 2008.

[22] T. C. Smith, I. G. Jacobson, B. Smith, T. I. Hooper, and M. A. K. Ryan, "The occupational role of women in military service: validation of occupation and prevalence of exposures in the Millennium Cohort Study," International Journal of Environmental Health Research, vol. 17, no. 4, pp. 271-284, 2007.

[23] M. A. K. Ryan, T. C. Smith, B. Smith et al., "Millennium Cohort: enrollment begins a 21-year contribution to understanding the impact of military service," Journal of Clinical Epidemiology, vol. 60, no. 2, pp. 181-191, 2007. 
[24] T. C. Smith, "The US Department of Defense Millennium Cohort Study: career Span and beyond longitudinal followup," Journal of Occupational and Environmental Medicine, vol. 51, no. 10, pp. 1193-1201, 2009.

[25] T. C. Smith, B. Smith, T. E. Corbeil, J. R. Riddle, and M. A. K. Ryan, "Self-reported mental health among US military personnel prior and subsequent to the terrorist attacks of September 11, 2001," Journal of Occupational and Environmental Medicine, vol. 46, no. 8, pp. 775-782, 2004.

[26] L. E. Kazis, A. Lee, A. Spiro III et al., "Measurement comparisons of the medical outcomes study and veterans SF36 health survey," Health Care Financing Review, vol. 25, no. 4, pp. 43-58, 2004.

[27] L. F. Kazis, D. R. Miller, J. A. Clark et al., "Improving the response choices on the veterans SF-36 health survey role functioning scales: results from the veterans health study," Journal of Ambulatory Care Management, vol. 27, no. 3, pp. 263-280, 2004.

[28] L. E. Kazis, D. R. Miller, K. M. Skinner et al., "Patient-reported measures of health: the veterans health study," Journal of Ambulatory Care Management, vol. 27, no. 1, pp. 70-83, 2004.

[29] J. E. Ware, M. Kosinski, and S. D. Keller, SF-36 Physical and Mental Health Summary Scales: A User's Manual, Health Assessment Lab, Boston, Mass, USA, 1994.

[30] J. E. Ware, M. Kosinski, and B. Gandek, SF-36 Health Survey: Manual \& Interpretation Guide, QualityMetric Incorporated, Lincoln, UK, 2005.

[31] J. E. Ware and C. D. Sherbourne, "The MOS 36-item shortform health survey (SF-36): I. Conceptual framework and item selection," Medical Care, vol. 30, no. 6, pp. 473-483, 1992.

[32] S. Dhalla and J. A. Kopec, "The CAGE questionnaire for alcohol misuse: a review of reliability and validity studies," Clinical and Investigative Medicine, vol. 30, no. 1, pp. 33-41, 2007.

[33] J. A. Ewing, "Detecting alcoholism. The CAGE questionnaire," Journal of the American Medical Association, vol. 252, no. 14, pp. 1905-1907, 1984.

[34] K. Y. Liang and S. L. Zeger, "Longitudinal data analysis using generalized linear models," Biometrika, vol. 73, no. 1, pp. 1322, 1986.

[35] S. L. Zeger and K. Y. Liang, "Longitudinal data analysis for discrete and continuous outcomes," Biometrics, vol. 42, no. 1, pp. 121-130, 1986.

[36] G. Maldonado and S. Greenland, "Simulation study of confounder-selection strategies," American Journal of Epidemiology, vol. 138, no. 11, pp. 923-936, 1993.

[37] J. R. Riddle, T. C. Smith, B. Smith et al., "Millennium Cohort: the 2001-2003 baseline prevalence of mental disorders in the U.S. military," Journal of Clinical Epidemiology, vol. 60, no. 2, pp. 192-201, 2007.

[38] T. C. Smith, M. Zamorski, B. Smith et al., "The physical and mental health of a large military cohort: baseline functional health status of the Millennium Cohort," BMC Public Health, vol. 7, article 340, 2007.

[39] F. Eskandari, J. I. Webster, and E. M. Sternberg, "Neural immune pathways and their connection to inflammatory diseases," Arthritis Research and Therapy, vol. 5, no. 6, pp. 251265,2003

[40] M. N. Silverman and E. M. Sternberg, "Neuroendocrineimmune interactions in rheumatoid arthritis: mechanisms of glucocorticoid resistance," NeuroImmunoModulation, vol. 15, no. 1, pp. 19-28, 2008.
[41] N. Danchenko, J. A. Satia, and M. S. Anthony, "Epidemiology of systemic lupus erythematosus: a comparison of worldwide disease burden," Lupus, vol. 15, no. 5, pp. 308-318, 2006.

[42] S. E. Gabriel and K. Michaud, "Epidemiological studies in incidence, prevalence, mortality, and comorbidity of the rheumatic diseases," Arthritis Research and Therapy, vol. 11, no. 3, pp. 229-244, 2009.

[43] D. J. McCarty, S. Manzi, T. A. Medsger Jr., R. RamseyGoldman, R. E. LaPorte, and C. K. Kwoh, "Incidence of systemic lupus erythematosus: race and gender differences," Arthritis and Rheumatism, vol. 38, no. 9, pp. 1260-1270, 1995.

[44] D. Fairweather and N. R. Rose, "Women and autoimmune diseases," Emerging Infectious Diseases, vol. 10, no. 11, pp. 2005-2011, 2004.

[45] F. Wolfe, K. Michaud, T. Li, and R. S. Katz, "EQ-5D and SF-36 quality of life measures in systemic lupus erythematosus: comparisons with rheumatoid arthritis, noninflammatory rheumatic disorders, and fibromyalgia," Journal of Rheumatology, vol. 37, no. 2, pp. 296-304, 2009.

[46] T. Uhlig, J. H. Loge, I. S. Kristiansen, and T. K. Kvien, "Quantification of reduced health-related quality of life in patients with rheumatoid arthritis compared to the general population," Journal of Rheumatology, vol. 34, no. 6, pp. 1241-1247, 2007.

[47] K. P. Liao, L. Alfredsson, and E. W. Karlson, "Environmental influences on risk for rheumatoid arthritis," Current Opinion in Rheumatology, vol. 21, no. 3, pp. 279-283, 2009.

[48] J. E. Oliver and A. J. Silman, "What epidemiology has told us about risk factors and aetiopathogenesis in rheumatic diseases," Arthritis Research and Therapy, vol. 11, no. 3, pp. 223-234, 2009.

[49] R. F. J. Cleveland and J. L. Ulcek, "Questions and answers about biological effects and potential hazards of radiofrequency electromagnetic fields," in Federal Communications Commission Office of Engineering \& Technology, OET Bulletin 56, Ed., Washington, DC, USA, 1999.

[50] J. P. Chretien, L. K. Chu, T. C. Smith et al., "Demographic and occupational predictors of early response to a mailed invitation to enroll in a longitudinal health study," BMC Medical Research Methodology, vol. 7, pp. 6-15, 2007.

[51] C. A. LeardMann, B. Smith, T. C. Smith, T. S. Wells, and M. A. K. Ryan, "Smallpox vaccination: comparison of self-reported and electronic vaccine records in the millennium cohort study," Human Vaccines, vol. 3, no. 6, pp. 245-251, 2007.

[52] B. Smith, L. K. Chu, T. C. Smith et al., "Challenges of selfreported medical conditions and electronic medical records among members of a large military cohort," BMC Medical Research Methodology, vol. 8, pp. 37-47, 2008.

[53] B. Smith, C. A. Leard, T. C. Smith, R. J. Reed, and M. A. K. Ryan, "Anthrax vaccination in the Millennium Cohort: validation and measures of health," American Journal of Preventive Medicine, vol. 32, no. 4, pp. 347-353, 2007.

[54] B. Smith, T. C. Smith, G. C. Gray, and M. A. K. Ryan, "When epidemiology meets the internet: web-based surveys in the millennium cohort study," American Journal of Epidemiology, vol. 166, no. 11, pp. 1345-1354, 2007.

[55] B. Smith, D. L. Wingard, M. A. K. Ryan, C. A. Macera, T. L. Patterson, and D. J. Slymen, "U.S. military deployment during 2001-2006: comparison of subjective and objective data sources in a large prospective health study," Annals of Epidemiology, vol. 17, no. 12, pp. 976-982, 2007. 
[56] T. C. Smith, B. Smith, I. G. Jacobson, T. E. Corbeil, and M. A. K. Ryan, "Reliability of standard health assessment instruments in a large, population-based cohort study," Annals of Epidemiology, vol. 17, no. 7, pp. 525-532, 2007.

[57] T. S. Wells, I. G. Jacobson, T. C. Smith et al., "Prior health care utilization as a potential determinant of enrollment in a 21year prospective study, the Millennium Cohort Study," European Journal of Epidemiology, vol. 23, no. 2, pp. 79-87, 2008.

[58] J. H. Barlow, A. P. Turner, and C. C. Wright, "Comparison of clinical and self-reported diagnoses for participants on a community-based arthritis self-management programme," British Journal of Rheumatology, vol. 37, no. 9, pp. 985-987, 1998.

[59] M. C. Hochberg, D. L. Perlmutter, T. A. Medsger et al., "Prevalence of self-reported physician-diagnosed systemic lupus erythematosus in the USA," Lupus, vol. 4, no. 6, pp. 454-456, 1995.

[60] E. W. Karlson, I. M. Lee, N. R. Cook, J. E. Manson, J. E. Buring, and C. H. Hennekens, "Comparison of self-reported diagnosis of connective tissue disease with medical records in female health professionals. The women's health cohort study," American Journal of Epidemiology, vol. 150, no. 6, pp. 652-660, 1999.

[61] I. Rasooly, A. C. Papageorgiou, and E. M. Badley, "Comparison of clinical and self reported diagnosis for rheumatology outpatients," Annals of the Rheumatic Diseases, vol. 54, no. 10, pp. 850-852, 1995.

[62] V. L. Star, J. C. Scott, R. Sherwin, N. Lane, M. C. Nevitt, and M. C. Hochberg, "Validity of self-reported rheumatoid arthritis in elderly women," Journal of Rheumatology, vol. 23, no. 11, pp. 1862-1865, 1996.

[63] B. T. Walitt, F. Constantinescu, J. D. Katz et al., "Validation of self-report of rheumatoid arthritis and systemic lupus erythematosus: the women's health initiative," Journal of Rheumatology, vol. 35, no. 5, pp. 811-818, 2008. 


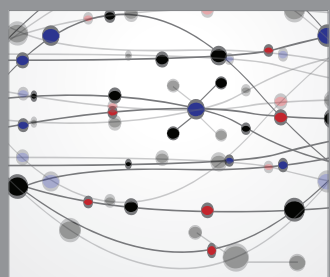

The Scientific World Journal
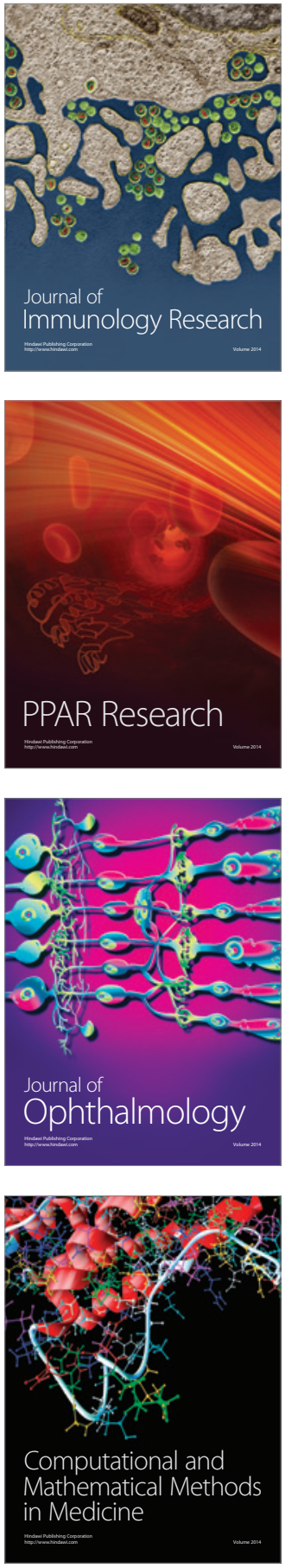

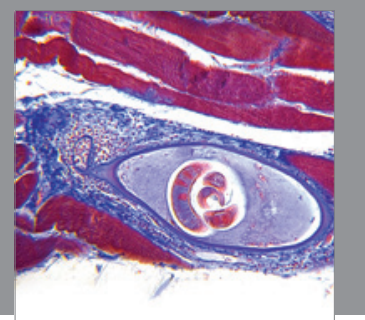

Gastroenterology

Research and Practice
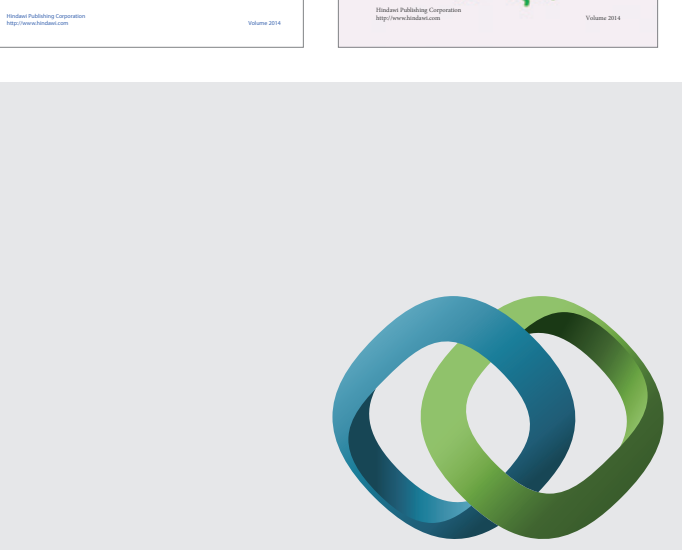

\section{Hindawi}

Submit your manuscripts at

http://www.hindawi.com
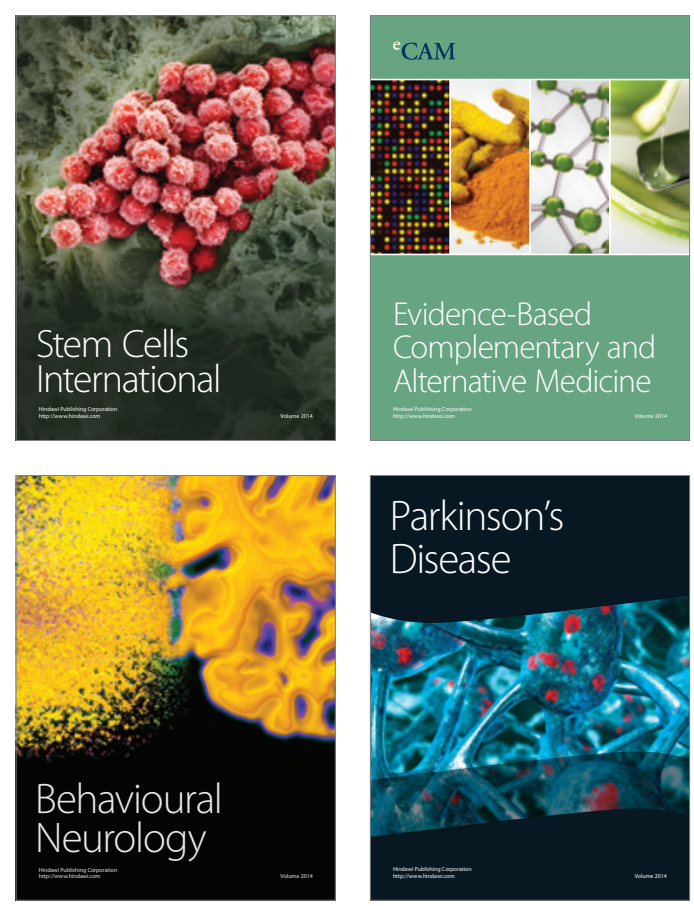

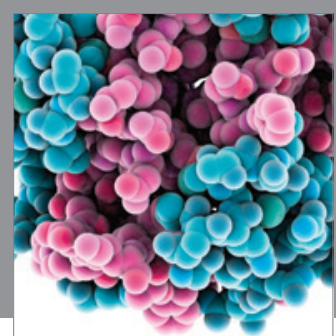

Journal of
Diabetes Research

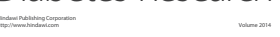

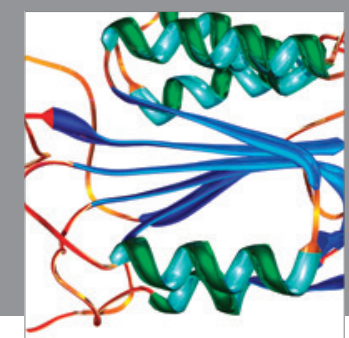

Disease Markers
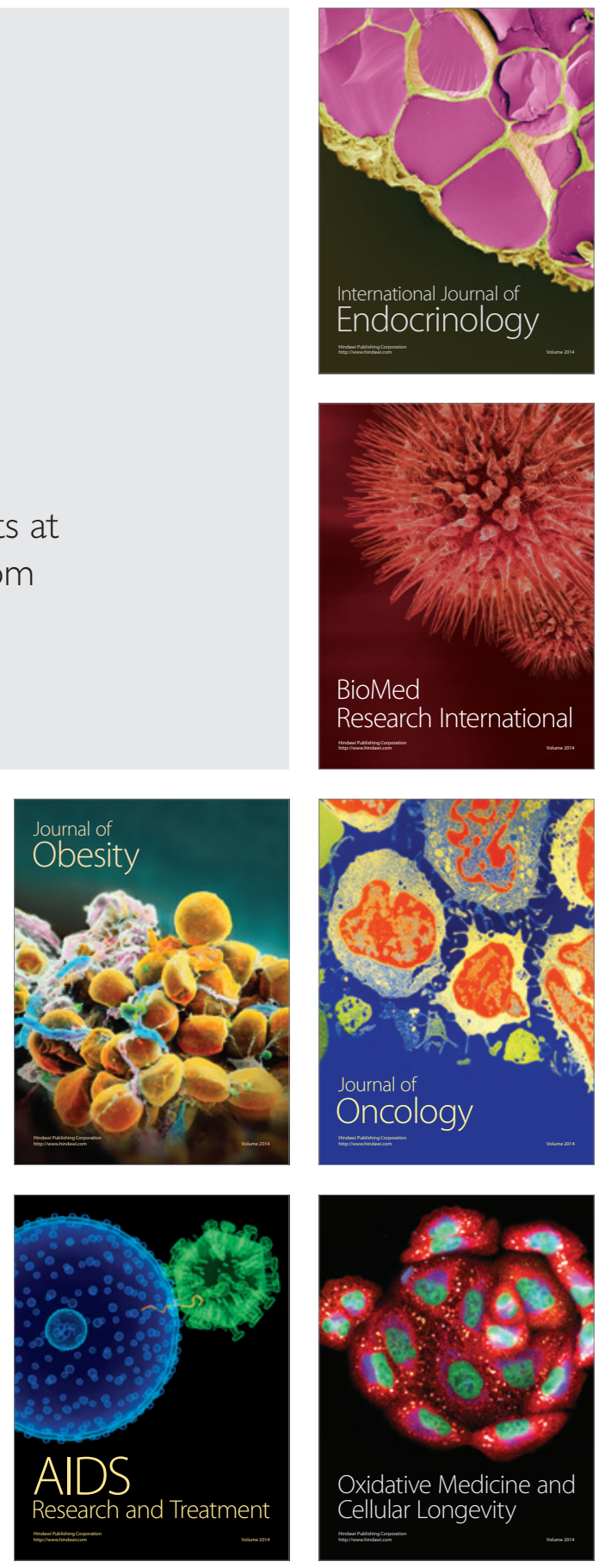